\title{
Potential biomarkers Ang II/AT1R and S1P/S1PR1 predict the prognosis of hepatocellular carcinoma
}

\author{
YUANYUAN JI ${ }^{1}$, HAIYAN CHEN ${ }^{1}$, WEI GOW ${ }^{2}$, LI MA ${ }^{3}$, YAOFENG JIN ${ }^{3}$, \\ BO HUI ${ }^{4}$, ZHENGAN YANG $^{4}$ and ZHIDONG WANG ${ }^{4}$ \\ ${ }^{1}$ Scientific Research Center and Precision Medical Institute, The Second Affiliated Hospital, Xi'an Jiaotong \\ University, Xi'an, Shaanxi 710004; ${ }^{2}$ Basic Medical Experiment Teaching Center, Health Science Center, \\ Xi'an Jiaotong University, Xi'an, Shaanxi 710061; Departments of ${ }^{3}$ Pathology and ${ }^{4}$ General Surgery, \\ The Second Affiliated Hospital, Xi'an Jiaotong University, Xi'an, Shaanxi 710004, P.R. China
}

Received February 19, 2020; Accepted July 7, 2020

DOI: $10.3892 / \mathrm{ol} .2020 .12071$

\begin{abstract}
Hepatocellular carcinoma (HCC) is one of the major causes of cancer-associated morbidity and mortality worldwide. Sphingosine-1-phosphate (S1P) and S1P receptor 1 (S1PR1) have been associated with the development and progression of HCC. Angiotensin II (Ang II) and Ang II receptor type 1 (AT1R) serve key roles in the progression and metastasis of HCC. However, the association and roles of Ang II/AT1R and S1P/S1PR1 in HCC have remained elusive. Therefore, the aim of the present study was to investigate the potential association between Ang II/AT1R and S1P/S1PR1 in HCC, as well as the association of AT1R and S1PR1 protein expression levels with the progression and prognosis of HCC. The results found that the serum levels of Ang II and S1P were significantly higher in patients with HCC compared with those in healthy donors. Furthermore, mRNA and protein levels of AT1R and S1PR1 were highly expressed in human HCC tissues. In addition, a positive correlation between Ang II/S1P and AT1R/S1PR1 in HCC was noted. Upregulation of AT1R and S1PR1 was associated with the progression of HCC. Patients with high AT1R and S1PR1 protein expression levels had unfavorable outcomes with respect to overall survival and recurrence-free survival compared with patients with low AT1R and S1PR1 expression levels. The present results demonstrated an association between AT1R and S1PR1 overexpression and the progression of $\mathrm{HCC}$, indicating that
\end{abstract}

Correspondence to: Dr Zhidong Wang, Department of General Surgery, The Second Affiliated Hospital, Xi'an Jiaotong University, 157 West 5th Road, Xi'an, Shaanxi 710004, P.R. China

E-mail: xawzd@163.com

Abbreviations: Ang II, angiotensin II; AT1R, Ang II receptor type 1; OS, overall survival; RAS, renin-angiotensin system; RFS, recurrence-free survival

Key words: hepatocellular carcinoma, sphingosine1-phosphate, prognosis, biomarkers
Ang II/AT1R and S1P/S1PR may serve as valuable prognostic biomarkers for HCC.

\section{Introduction}

Hepatocellular carcinoma (HCC) is one of the most prevalent types of malignancy worldwide, with a rich blood supply and high levels of metastasis and recurrence $(1,2)$. The overall 5-year survival rate for HCC is $\sim 30 \%$ (3). HCC has been found to invade blood vessels, resulting in intra- and extrahepatic metastases (4). At present, hepatectomy and liver transplantation are key treatments with curative potential in patients with HCC (5). However, the overall survival (OS) rate of patients with $\mathrm{HCC}$ remains unsatisfactory due to the high incidence of tumor recurrence and metastasis (6). Thus, there is a requirement to identify novel biomarkers for the progression of HCC to enhance the OS rate of patients.

Sphingosine-1-phosphate (S1P) is a vital metabolite that serves a key role in intra- and intercellular signal transduction (7). S1P regulates multiple cellular functions, such as cell proliferation, migration, adhesion and inflammation, via high-affinity G protein-coupled S1P receptors (S1PRs) (8), which affect cellular activities by inhibiting cell apoptosis and promoting cell proliferation (9). Furthermore, activation of S1PR1 is involved in the regulation of numerous cell behaviors associated with aggressiveness and cancer progression, including tumor growth, invasion and migration $(10,11)$. Previous studies have also found that S1P and its receptors are implicated in the progression of $\operatorname{HCC}(12,13)$.

The function of the renin-angiotensin system (RAS) in tumor invasion or metastasis has previously been reported (14). High protein and mRNA expression levels of RAS components in human pancreatic cancer tissues have been associated with tumor grade and clinical prognosis (15). Angiotensin II (Ang II), an important peptide of RAS, exerts its effects by activating Ang II receptor type 1 (AT1R) and AT2R. AT1R has been found to be involved in the majority of essential physiological actions, such as blood pressure and sodium balance, and pathophysiological actions, such as inflammation and angiogenesis, of Ang II $(16,17)$. Ang II and AT1R have essential roles in tumor survival, angiogenesis and proliferation 
in all types of cancer (18), and have also been associated with the progression and metastasis of HCC (19-21).

Ang II induces proliferation of smooth muscle cells by the S1P signaling pathway (16). Ang II is also able to augment S1PR1 protein and mRNA expression levels and migration of rat aortic smooth muscle cells, which can be inhibited by treatment with antagonists of AT1R and S1PR1 (22). However, the association and roles of Ang II/AT1R and S1P/S1PR1 in $\mathrm{HCC}$ have remained largely elusive. Therefore, the aim of the present study was to investigate the association between AT1R and S1PR1 mRNA and protein expression levels and the clinicopathological characteristics of patients with HCC, as well as the progression of HCC. Additionally, the utility of these potential biomarkers in the prediction of prognosis in affected patients was assessed.

\section{Materials and methods}

Patients and tissue samples. A total of 75 patients with HCC (51 males and 24 females) who underwent resection of HCC at the Second Affiliated Hospital of Xi'an Jiaotong University (Xi'an, China) between January 2013 and January 2017 were included in the present retrospective study. Data was also collected from the medical files of the patients. Liver cancer and adjacent normal tissues ( $\geq 5 \mathrm{~cm}$ from the tumor) were collected from the 75 patients and frozen at $-180^{\circ} \mathrm{C}$ for further analysis. Serum samples $(n=75)$ were collected from $4 \mathrm{ml}$ peripheral blood and stored at $4^{\circ} \mathrm{C}$ in a 5-ml tube. After centrifugation at $3,000 \times \mathrm{g}$ for $10 \mathrm{~min}$ at $4^{\circ} \mathrm{C}$, the obtained serum $(1 \mathrm{ml})$ was stored at $-80^{\circ} \mathrm{C}$. Serum samples from the control group were collected from 66 healthy subjects without any disease, matched according to sex and age. The patients did not receive any chemotherapy, radiotherapy or immunotherapy prior to surgery. The liver cancer and adjacent normal tissues were collected for immunohistochemical analysis. According to the Ethics Committee of the Second Affiliated Hospital of Xi'an Jiaotong University (Xi'an, China), all participants provided written informed consent. Clinical data (including age, sex, stage, tumor size, intrahepatic metastasis, portal vein invasion and Edmondson grade) were collected from the medical records of each patient (Table I). All the specimens were graded using the Edmonson method (23). According to the differentiation degree of tumor cells, HCC tissues were categorized into grades I-III. Grade I was defined as low-grade HCC, grade II as medium-grade HCC and grade III as high-grade HCC. Furthermore, the TNM stage was defined according to the American Joint Committee on Cancer TNM Staging for Liver Tumors as follows (24): TX, primary tumor cannot be assessed; T0, no evidence of primary tumor; T1, solitary tumor without vascular invasion; T2, solitary tumor with vascular invasion or multiple tumors $<5 \mathrm{~cm}$ in size; T3a, multiple tumors $>5 \mathrm{~cm}$ in size; T3b, single tumor or multiple tumors of any size, involving a major branch of the portal vein or hepatic vein; and T4, tumor(s) with direct invasion of adjacent organs, other than the gallbladder, or with perforation of visceral peritoneum.

The patients were followed up from January 2016 to January 2020, initially every 2 months and at least every 3-6 months following surgery. Furthermore, the liver function and serum $\alpha$-fetoprotein (AFP) levels were examined, and abdominal ultrasonography was performed. If recurrence was suspected, CT or MRI scan was performed immediately. OS and recurrence-free survival (RFS) times were defined as the interval between surgery and death or recurrence, respectively. If recurrence was not diagnosed, the patient was examined at the date of death or at the last follow-up evaluation. All of the aforementioned investigations were performed as previously described $(25,26)$.

ELISA. Serum samples were collected from the peripheral blood samples, centrifuged at $4,000 \mathrm{x}$ g for $10 \mathrm{~min}$ at $-4^{\circ} \mathrm{C}$ and then stored at $-80^{\circ} \mathrm{C}$ for further analysis. According to the manufacturer's instructions, Ang II (cat. no. ADI-900-204; Enzo Life Sciences, Inc.) and S1P levels (cat. no. abx585002; Abbexa Ltd.) were assessed using their respective ELISA kits. Each analysis was performed in triplicate.

Western blot analysis. As previously described (27), total protein was extracted using RIPA lysis buffer (cat. no. PP1901; BioTeke Corporation), and protein concentration was determined using a BCA protein detection kit (cat. no. P0010; Beyotime Institute of Biotechnology). Protein samples (25 $\mu \mathrm{g}$ /lane) were separated via 10\% SDS-PAGE and transferred onto PVDF membranes (Bio-Rad Laboratories, Inc.), then blocked with $5 \%$ skimmed milk in TBS buffer containing $0.1 \%$ Tween-20 at $25^{\circ} \mathrm{C}$ for $2 \mathrm{~h}$, and incubated with specific antibodies against AT1R (1:500; cat. no. ab124734), S1PR1 (1:500; cat. no. ab77076) and $\beta$-actin (1:1,000; cat. no. ab200658) at $-4^{\circ} \mathrm{C}$ overnight (all Abcam). $\beta$-actin was used as the internal control. Subsequently, membranes were incubated with an HRP-conjugated goat anti-rabbit IgG H\&L secondary antibody (1:5,000; cat. no. ab7090; Abcam) at $37^{\circ} \mathrm{C}$ for $1 \mathrm{~h}$. Enhanced chemiluminescence reagents (Pierce; Thermo Fisher Scientific, Inc.) were used to visualize the blots, and the optical densities of the bands were scanned and quantified using Syngene Gene Tools (Syngene Europe; model $\mathrm{G}$ box chem hr16; serial no. SYDR4/2327). A total of three independent experiments was performed.

Reverse transcription-quantitative $(R T-q) P C R$. mRNA levels were detected as previously described (27). Total RNA was extracted using the TRIzol ${ }^{\circledR}$ reagent (Invitrogen; Thermo Fisher Scientific, Inc.). cDNA was synthesized from total RNA $(1 \mu \mathrm{g})$ using a PrimeScript ${ }^{\mathrm{TM}} \mathrm{RT}$ master mix kit (Takara Bio, Inc.) according to the manufacturer's instructions. qPCR was performed using a SYBR Premix Ex Taq ${ }^{\mathrm{TM}}$ II Perfect Real-Time kit (Takara Bio, Inc.) on an ABI qPCR System (Applied Biosystems; Thermo Fisher Scientific, Inc.) according to the manufacturer's instructions. Each sample was run in triplicate. Primers for AT1R, S1PR1 and $\beta$-actin were designed using the Beacon designer 4.0 software (version 4.0; Premier Biosoft International). The primer sequences were as follows: AT1R forward, 5'-AGACTGGCCTTCTCTGGA-3' and reverse, 5'-CACCGAGGAATACGCTTT-3'; S1PR1 forward, 5'-CACGCTTTCTGTGGCTTGGA-3' and reverse, 5'-CGACGATGGCGCTCCAACA-3'; and $\beta$-actin forward, 5'-TGGCACCCAGCACAATGAA-3' and reverse, 5'-CTA AGTCATAGTCCGCCTAGAAGCA-3'. The RT-PCR products were observed using electrophoresis and $2 \%$ agarose gels with ethidium bromide. A melting point dissociation curve 
Table I. Association between AT1R and S1PR1 expression levels and pathological characteristics of patients with hepatocellular carcinoma.

\begin{tabular}{|c|c|c|c|c|c|c|c|}
\hline \multirow[b]{2}{*}{ Characteristic } & \multirow[b]{2}{*}{ Patients, $\mathrm{n}$} & \multicolumn{2}{|c|}{ AT1R expression } & \multirow[b]{2}{*}{ P-value } & \multicolumn{2}{|c|}{ S1PR1 expression } & \multirow[b]{2}{*}{ P-value } \\
\hline & & Low, n (\%) & High, n (\%) & & Low, n (\%) & High, n (\%) & \\
\hline Sex & & & & 0.134 & & & $>0.999$ \\
\hline Male & 51 & $19(37.3)$ & $32(62.7)$ & & $23(45.1)$ & $28(54.9)$ & \\
\hline Female & 24 & $14(58.3)$ & $10(41.7)$ & & $11(45.8)$ & $13(54.2)$ & \\
\hline Age, years & & & & 0.337 & & & 0.097 \\
\hline$\geq 50$ & 49 & $23(46.9)$ & $26(53.1)$ & & $20(40.8)$ & $29(59.2)$ & \\
\hline$<50$ & 26 & $9(34.6)$ & $17(65.4)$ & & $16(61.5)$ & $10(38.5)$ & \\
\hline Tumor size, $\mathrm{cm}$ & & & & 0.602 & & & 0.589 \\
\hline$\geq 4$ & 55 & $25(45.5)$ & $30(54.5)$ & & $17(30.9)$ & $38(69.1)$ & \\
\hline$<4$ & 20 & $11(55.0)$ & $9(45.0)$ & & $6(30.0)$ & $14(70.0)$ & \\
\hline Hepatitis B surface antigen & & & & $>0.999$ & & & 0.759 \\
\hline Positive & 61 & $28(45.9)$ & $33(54.1)$ & & $22(36.1)$ & $39(63.9)$ & \\
\hline Negative & 14 & $6(42.9)$ & $8(57.1)$ & & $4(28.6)$ & $10(71.4)$ & \\
\hline Serum $\alpha$-fetoprotein, $\mathrm{ng} / \mathrm{ml}$ & & & & 0.450 & & & $>0.999$ \\
\hline$\geq 400$ & 53 & $23(43.4)$ & $30(56.6)$ & & $20(37.7)$ & $33(62.3)$ & \\
\hline$<400$ & 22 & $12(54.5)$ & $10(45.5)$ & & $8(36.4)$ & $14(63.6)$ & \\
\hline Intrahepatic metastasis & & & & $0.017^{\mathrm{a}}$ & & & $0.008^{b}$ \\
\hline Yes & 48 & $16(33.3)$ & $32(66.7)$ & & $13(27.1)$ & $35(72.9)$ & \\
\hline No & 27 & $17(63.0)$ & $10(37.0)$ & & $16(59.3)$ & $11(40.7)$ & \\
\hline Portal vein invasion & & & & $0.033^{\mathrm{a}}$ & & & $0.015^{\mathrm{a}}$ \\
\hline Yes & 46 & $15(32.6)$ & $31(67.4)$ & & $12(26.1)$ & $34(73.9)$ & \\
\hline No & 29 & $17(58.6)$ & $12(41.4)$ & & $16(55.2)$ & $13(44.8)$ & \\
\hline Edmondson grade & & & & $0.017^{\mathrm{a}}$ & & & $0.008^{b}$ \\
\hline I & 13 & $8(61.5)$ & $5(38.5)$ & & $9(69.2)$ & $4(30.8)$ & \\
\hline II & 40 & $15(37.5)$ & $25(62.5)$ & & $13(32.5)$ & $27(67.5)$ & \\
\hline III & 22 & $12(54.5)$ & $10(45.5)$ & & $15(68.2)$ & $7(31.8)$ & \\
\hline TNM stage & & & & $0.004^{\mathrm{b}}$ & & & $0.015^{\mathrm{a}}$ \\
\hline I-II & 48 & $18(37.5)$ & $30(62.5)$ & & $23(47.9)$ & $25(52.1)$ & \\
\hline III-IV & 27 & $20(74.1)$ & $7(25.9)$ & & $21(77.8)$ & $6(22.2)$ & \\
\hline
\end{tabular}

${ }^{\mathrm{a}} \mathrm{P}<0.05 ;{ }^{\mathrm{b}} \mathrm{P}<0.01$. AT1R, angiotensin II receptor type 1; S1PR1, sphingosine-1-phosphate receptor 1.

was used to indicate that only a single product was shown. For qPCR, relative mRNA levels were analyzed using the $2^{-\Delta \Delta C t}$ method (28). Data were standardized to $\beta$-actin mRNA levels. A total of three independent experiments were performed.

Immunohistochemistry. Liver cancer tissue samples were collected from 75 patients with HCC following hepatic resection at the Second Affiliated Hospital of Xi'an Jiaotong University between January 2013 and January 2017. During the same period, adjacent normal liver tissue samples were also obtained. All specimens (adjacent normal and liver cancer tissues) were investigated using immunohistochemistry. Tissue samples were fixed in $10 \%$ formalin at room temperature for $48 \mathrm{~h}$ and embedded in paraffin. Next, $5-\mu \mathrm{m}$ sections were deparaffinized for antigen retrieval in citric acid buffer $(\mathrm{pH} 6.0$; $95^{\circ} \mathrm{C}$ for $20 \mathrm{~min}$ ). Sections were washed 3 times with PBS for 3 min each, 3 times with xylene for 5 min each, 2 times with
$100 \%$ ethanol for 10 min each, 2 times with $95 \%$ ethanol for 10 min each and finally 2 times with double-distilled water for 5 min each. Slides were treated with $3 \%$ hydrogen peroxide at room temperature for $10 \mathrm{~min}$ to block endogenous peroxidase activity. After blocking in 1\% BSA (Sigma-Aldrich; Merck $\mathrm{KGaA}$ ) at room temperature for $20 \mathrm{~min}$, the slides were incubated with rabbit anti-human AT1R (1:50; cat. no. ab124734; Abcam) and S1PR1 (1:100; cat. no. ab77076; Abcam) antibodies overnight at $4^{\circ} \mathrm{C}$. Subsequently, the slides were incubated with $2 \mu \mathrm{g} / \mathrm{ml}$ biotinylated anti-rabbit IgG secondary antibody (XianFeng Bioengineering Institute) at room temperature for $40 \mathrm{~min}$. Next, the sections were stained using a Standard Ultra-Sensitive ABC Peroxidase Staining kit (Thermo Fisher Scientific, Inc.), incubating the tissue sections with the ABC Reagent (Reagent A, $2 \mathrm{ml}$; Reagent B, $2 \mathrm{ml}$ ) at room temperature for $30 \mathrm{~min}$. Bright-field images (magnification, x200 and $\mathrm{x} 400$ ) were captured using an Axio Scan Z1 light microscope 

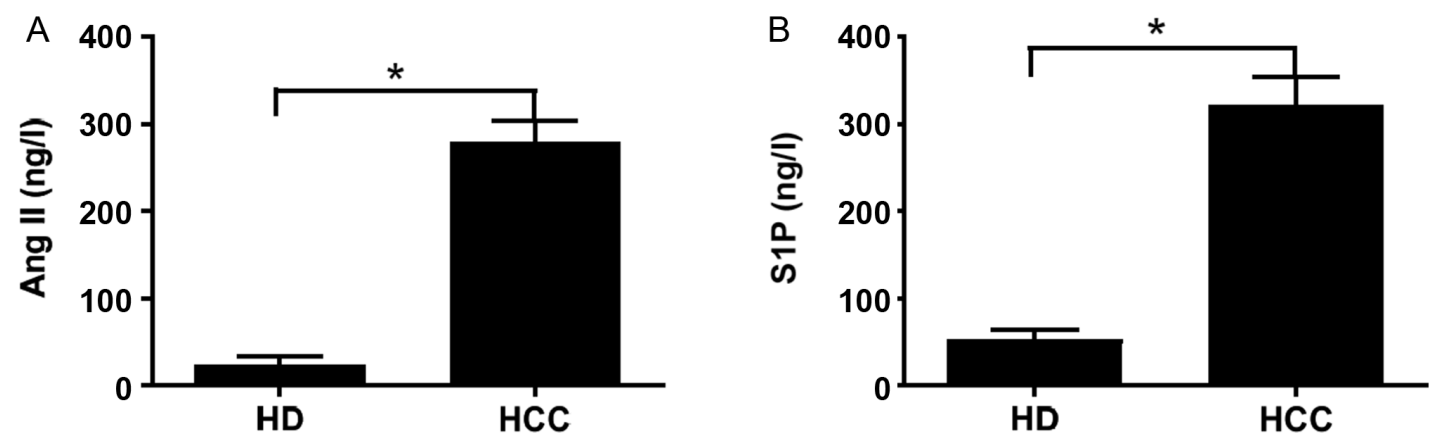

Figure 1. (A) Ang II and (B) S1P levels in healthy donors and patients with HCC. "P<0.05 vs. HD using an unpaired Student's t-test. Ang II, angiotensin II; S1P1, sphingosine1-phosphate 1; HD, healthy donors; HCC, hepatocellular carcinoma.

(Zeiss $\mathrm{GmbH}$ ). Immunostaining results were independently observed and interpreted by two pathologists blinded to the clinical data. Weak and strong staining were distinguished via the color of brown-yellow granules. When the brown-yellow granules were dark in the HCC tissues, this was considered as strong staining (high expression), while when the brown-yellow granules were weak in the HCC tissues, this was considered as weak staining (low expression).

Hepatitis B surface antigen (HBsAg) quantification. Quantification of HBsAg was performed using an automated chemiluminescent microparticle immunoassay using the ARCHITECT i2000 system (Abbott Pharmaceutical Co., Ltd.). All procedures were performed according to the manufacturer's protocol (29). The sensitivity and the specificity of this assay are 100 and $99.76 \%$, respectively, according to the manufacturer's protocol.

Statistical analysis. Data are expressed as the mean \pm standard deviation. A Fisher's exact test was performed to determine whether AT1R and S1PR1 expression levels in HCC were associated with clinicopathological characteristics. A two-tailed paired or unpaired Student's t-test was used to determine significant differences between groups. The Kaplan-Meier method was used for survival analysis, and differences in survival were investigated using the log-rank test. These statistical analyses were performed using SPSS software (version 19.0; IBM Corp.). Correlation between Ang II and S1P, and between AT1R and S1PR1 in patients with HCC were analyzed using Pearson's correlation coefficient (GraphPad Prism 8; GraphPad Software, Inc.). $\mathrm{P}<0.05$ was considered to indicate a statistically significant difference.

\section{Results}

ATIR and S1PRl expression levels in HCC tissue are associated with clinicopathological characteristics. The clinical characteristics and laboratory parameters of patients with HCC are summarized in Table I. The results indicated that AT1R and S1PR1 expression levels were not significantly associated with patient sex, age, levels of HBsAg or serum AFP, or tumor size. However, AT1R and S1PR1 expression levels in $\mathrm{HCC}$ were associated with intrahepatic metastasis $(\mathrm{P}=0.017$ and $\mathrm{P}=0.008$, respectively), portal vein invasion $(\mathrm{P}=0.033$ and $\mathrm{P}=0.015$, respectively), Edmondson grade $(\mathrm{P}=0.017$ and
$\mathrm{P}=0.008$, respectively) and TNM stage $(\mathrm{P}=0.004$ and $\mathrm{P}=0.015$, respectively).

Ang II and SIP levels are elevated in the serum of patients with HCC. The Ang II and S1P levels in the serum collected from the 66 healthy donors and 75 patients with HCC were determined, and the results revealed that both the serum levels were significantly higher in patients with HCC compared with those in healthy donors (Fig. 1).

ATIR and S1PRI are upregulated in HCC tissue. Next, AT1R and S1PR1 protein expression levels were investigated in HCC tissues from 75 patients using immunohistochemistry. Positive staining for AT1R and S1PR1 was displayed as brown-yellow granules, which were primarily located in the cell membrane or cytoplasm. The cells with positive staining exhibited a clear tissue cell structure, where uniform brown-yellow granules were present in the cell membrane or cytoplasm and staining was markedly higher compared with that in the background. In human normal liver tissue, AT1R and S1PR1 were weakly expressed, whereas they were strongly expressed in human HCC tissue (Fig. 2).

Protein and $m R N A$ expression levels of ATIR and SIPRI are elevated in HCC tissues. To further assess AT1R and S1PR1 protein and mRNA expression levels in HCC, liver cancer and adjacent normal tissue obtained from 3 representative samples (according to IHC staining) among the 75 HCC samples were subjected to western blot and RT-qPCR analyses. AT1R and S1PR1 protein expression levels in HCC tissue were significantly higher compared with those in normal liver tissue $(\mathrm{P}<0.05$; Fig. 3A and $\mathrm{B})$. In addition, RT-qPCR was performed to quantify AT1R and S1PR1 mRNA expression levels in HCC tissues. AT1R and S1PR1 mRNA expression levels in HCC tissue were significantly higher compared with those in normal liver tissue $(\mathrm{P}<0.05$; Fig. $3 \mathrm{C}$ and $\mathrm{D})$, which was consistent with western blot analysis results. In summary, western blot, RT-qPCR and immunohistochemical analyses demonstrated that AT1R and S1PR1 expression was increased in HCC tissue.

Significant positive correlation between Ang II and SIP and between ATIR and SIPRI in patients with HCC. The serum levels of Ang II and S1P in 66 healthy donors and 75 patients with $\mathrm{HCC}$ were determined using ELISA. A positive 


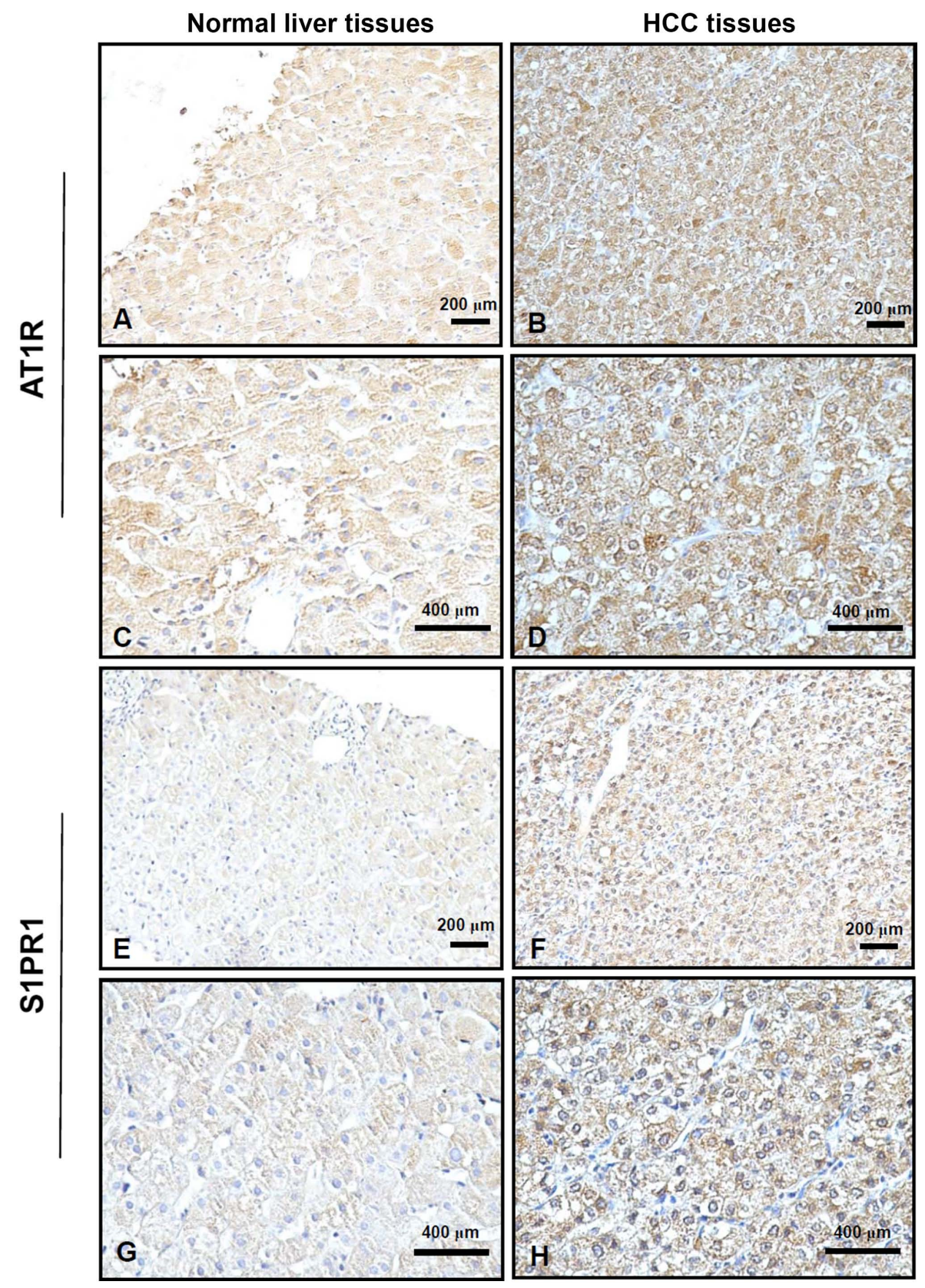

Figure 2. Differential protein expression levels of AT1R and S1PR1 in adjacent normal liver and human HCC tissues. (A) Weak AT1R staining in normal liver and (B) strong AT1R staining in HCC tissue (magnification, x200). (C) Weak AT1R staining in normal liver and (D) strong AT1R staining in HCC tissue (magnification, x400). (E) Weak S1PR1 staining in normal liver and (F) strong S1PR1 staining in HCC (magnification, x200). (G) Weak S1PR1 staining in normal liver and $(\mathrm{H})$ strong S1PR1 staining in HCC tissue (magnification, x400). AT1R, angiotensin II receptor type 1; S1PR1, sphingosine-1-phosphate receptor 1; HCC, hepatocellular carcinoma.

correlation was identified between Ang II and S1P in patients with HCC $(\mathrm{P}=0.002)$, but not in healthy donors $(\mathrm{P}=0.228$; Fig. 4A and B). Furthermore, the correlation between AT1R and S1PR1 protein expression levels in the $75 \mathrm{HCC}$ and normal adjacent tissues was also investigated and there was no correlation between AT1R and S1PR1 levels in the normal liver samples $(\mathrm{P}=0.081)$; however, there was a correlation in $\mathrm{HCC}$ tissue samples ( $\mathrm{P}=0.001$; Fig. $4 \mathrm{C}$ and $\mathrm{D})$.

ATIR and S1PRI protein levels are associated with prognosis of patients with HCC. Kaplan-Meier survival curves were used to determine the effects of AT1R and S1PR1 protein expression levels via immunohistochemical analyses on the prognosis of patients with HCC. The results revealed that OS and RFS rates were significantly lower in patients with high AT1R expression levels compared with those in patients with low AT1R levels $(\mathrm{P}=0.0250$ and $\mathrm{P}=0.0366$, respectively; Fig. 5A and B). The results also revealed that OS and RFS rates were significantly lower in patients with high S1PR1 expression levels compared with those in patients with low S1PR1 levels $(\mathrm{P}=0.0401$ and $\mathrm{P}=0.0359$, respectively; Fig. $5 \mathrm{C}$ and $\mathrm{D})$. The present results indicated that the 50-month survival rate of patients with HCC with high AT1R and S1PR1 expression was low. These results demonstrated that AT1R and S1PR1 upregulation was significantly associated with patient death and $\mathrm{HCC}$ recurrence. 
A

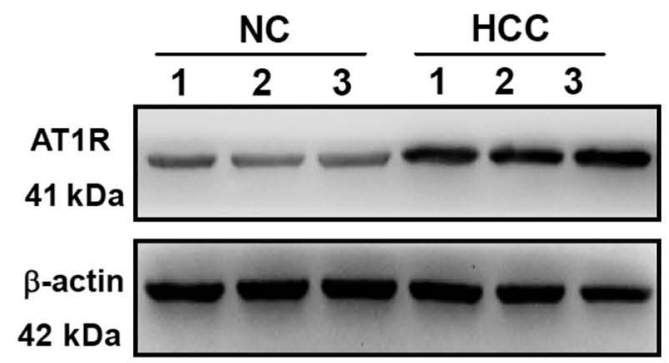

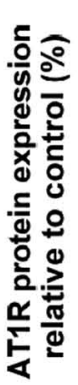

C
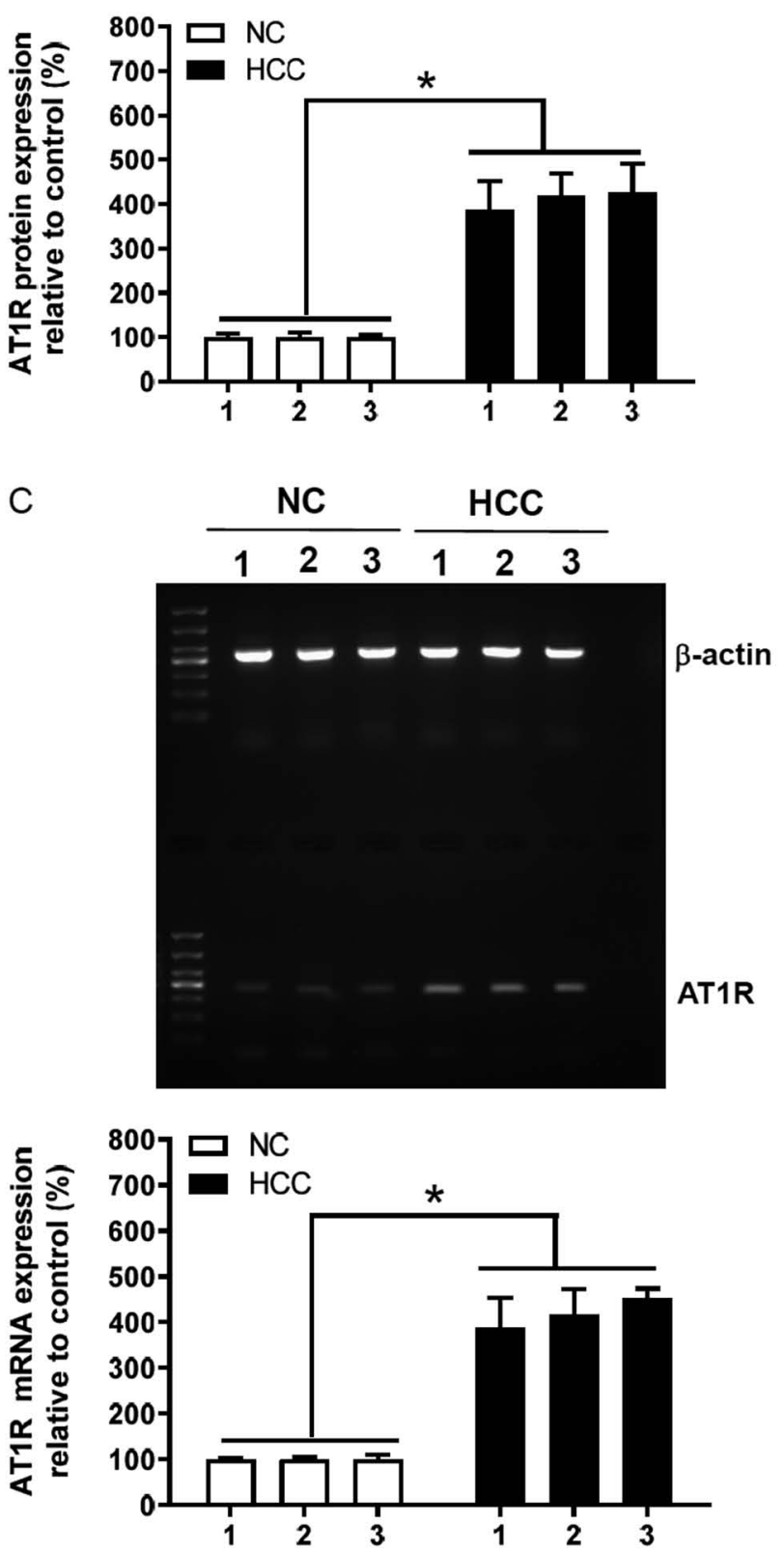

B

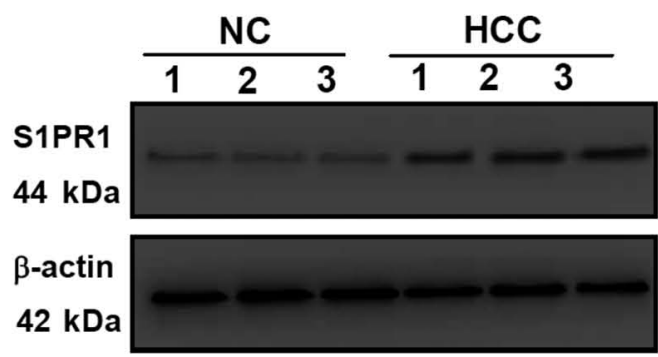

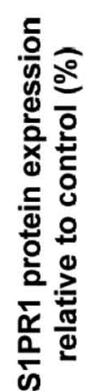

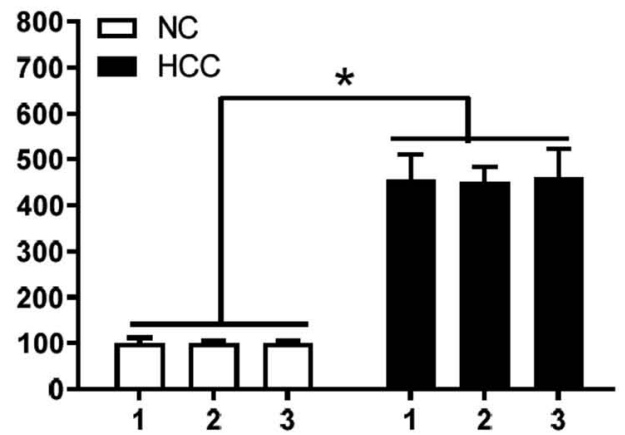

D
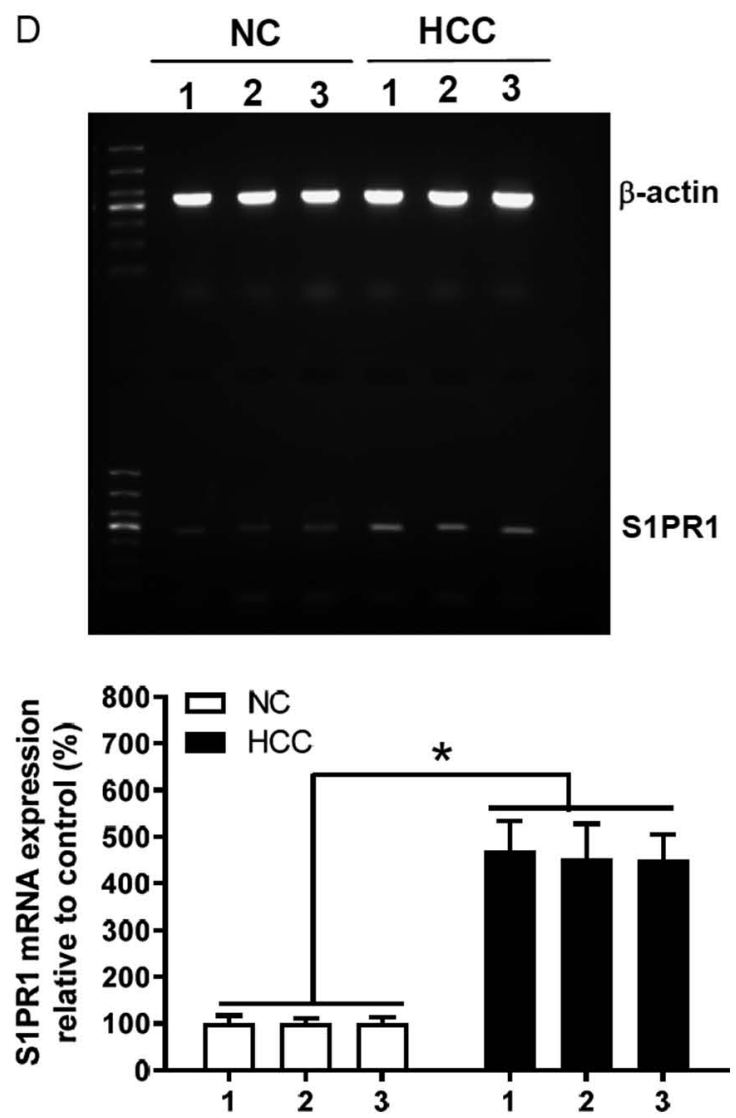

Figure 3. Protein and mRNA expression levels of AT1R and S1PR1 in adjacent normal liver and HCC tissues. Representative western blots showing protein expression levels of (A) AT1R and (B) S1PR1 in adjacent normal liver and HCC tissues. mRNA expression levels of (C) AT1R and (D) S1PR1 in adjacent normal liver and HCC tissues were determined using reverse transcription-quantitative PCR. Representative adjacent normal liver and HCC tissue samples were obtained from three different individuals. The means of the 3 samples for NC and HCC, respectively, were calculated and compared using a paired Student's t-test. ${ }^{*} \mathrm{P}<0.05$ vs. NC. AT1R, angiotensin II receptor type 1; S1PR1, sphingosine-1-phosphate receptor 1; HCC, hepatocellular carcinoma; NC, normal control.

\section{Discussion}

Evidence indicates that S1P and its receptors are associated with the pathophysiology of cancer and have a key role in cancer development (30). Elevated serum levels of S1P in patients with different types of cancer, including HCC, have been associated with a poor clinical prognosis $(12,13)$. S1P is a metabolite that serves a vital role in intra- and intercellular 

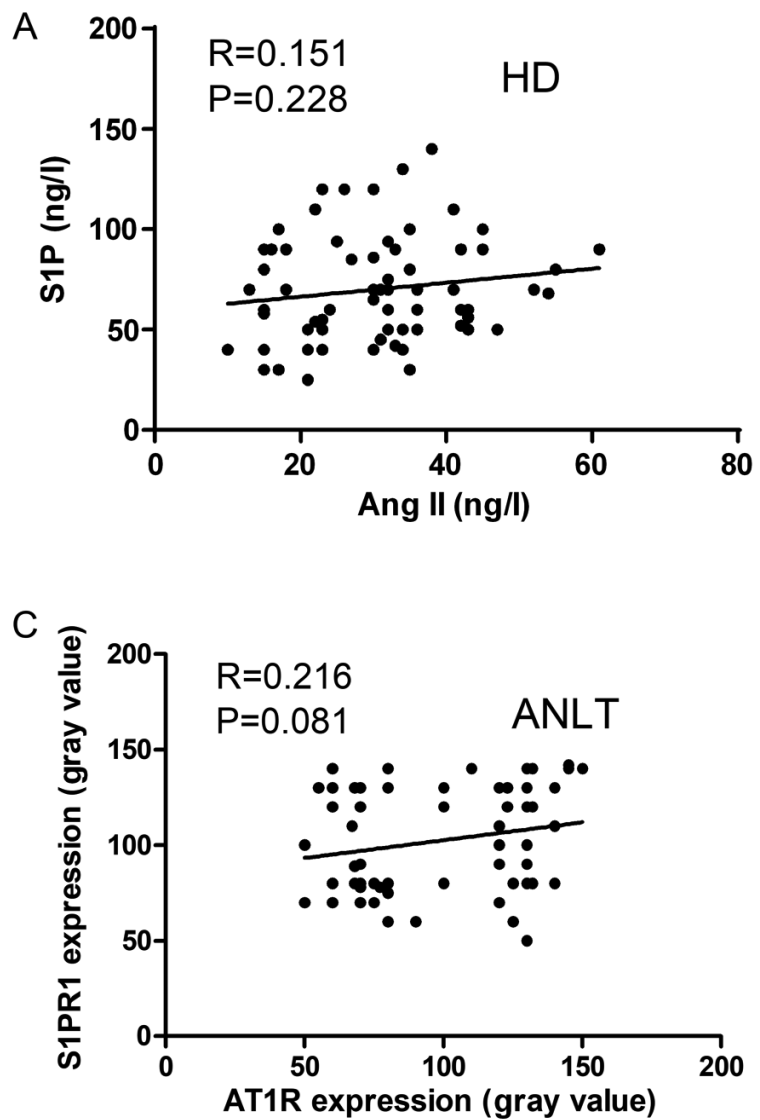
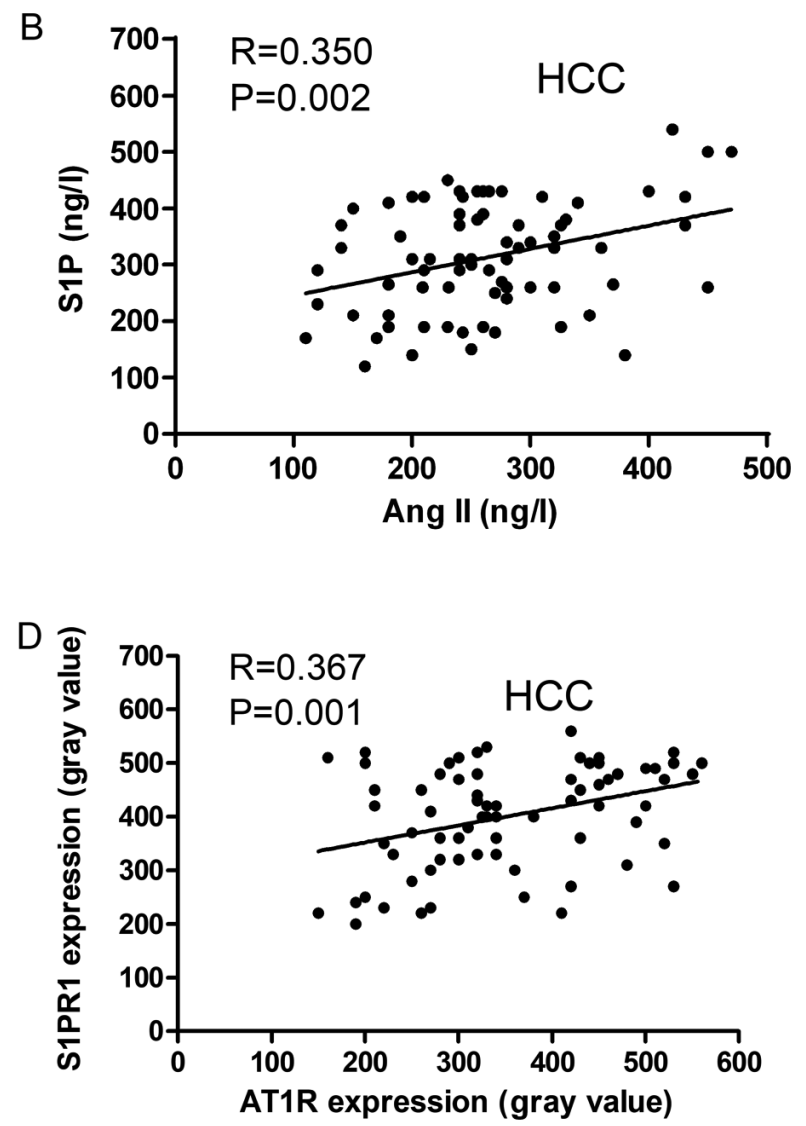

Figure 4. Correlation between Ang II and S1P, and AT1R and S1PR1 in HD ( $\mathrm{n}=66$ ) and patients with HCC ( $\mathrm{n}=75$ ). A correlation between Ang II and S1P levels was not observed in (A) HD, but was present in (B) patients with HCC. A correlation between AT1R and S1PR1 protein expression levels was not observed in (C) ANLT, but was present in (D) HCC tissues. Ang II and S1P levels were detected using ELISA. AT1R and S1PR1 protein expression levels were determined using western blot analysis. Ang II, angiotensin II; S1P, sphingosine-1-phosphate; AT1R, angiotensin II receptor type 1; S1PR1, sphingosine-1-phosphate receptor 1; HD, healthy donors; HCC, hepatocellular carcinoma; ANLT, adjacent normal liver tissues.

signal transduction (7). Previous studies have found that $\mathrm{S} 1 \mathrm{P}$ and its receptors are involved in the development of $\operatorname{HCC}(12,13)$. Consistent with the results from a previous study, the present study indicated that the serum S1P levels were significantly higher in patients with HCC compared with those in healthy donors (13). The present study inferred that the increased serum S1P levels may be due to changes in sphingolipid metabolism in liver cancer cells. Ang II, a central component of RAS, induces angiogenesis, proliferation and inflammation in HCC tissues via its primary receptor, AT1R (31). Furthermore, Ang II and AT1R are reported to be associated with tumor grade and clinical outcome in HCC (32). In addition its roles in cardiovascular disease and renal injury, Ang II has been associated with cell proliferation and inflammation, and may offer a potential target for the prevention and treatment of HCC (19). Consistent with previous studies $(32,33)$, the present study demonstrated that serum Ang II levels were significantly higher in patients with HCC compared with those in healthy donors. A previous study found that inflammation is associated with liver cancer (34). The present study speculated that Ang II stimulates angiogenesis, inflammation and proliferation in HCC tissue, which may increase the levels of active peptide Ang II. In the present study, Ang II and AT1R levels in the serum and liver tissue of patients with HCC, respectively, were significantly elevated. The present results suggested that Ang II and S1P levels may be associated with cell growth, migration and invasion, and with the prognosis of patients with HCC (Table I). However, it is not clear whether the increase of Ang II and S1P levels in the serum was consistent with that in HCC tissues.

Immunohistochemical analysis revealed that AT1R and S1PR1 were strongly expressed in human HCC tissue. In addition, AT1R and S1PR1 expression levels in patient tissue were assessed at the mRNA and protein levels using RT-qPCR and western blot analysis, respectively, which demonstrated that AT1R and S1PR1 mRNA and protein expression levels were increased in HCC tissue compared with those in normal adjacent liver tissue. $\mathrm{Xu}$ et al (33) found that Ang II protein expression levels were notably higher in human liver cancer tissue compared with those in normal adjacent tissues. Further investigations are required to assess protein and mRNA expression levels of Ang II and S1P in liver cancer tissue, as this was not performed in the present study. Notably, upregulation of AT1R and S1PR1 was associated with well-known cancer progression-associated clinicopathological parameters, including intrahepatic metastasis, portal vein invasion, histological differentiation and TNM stage in the present study, which indicates that upregulation of AT1R and S1PR1 may be a significant contributing factor in the progression of HCC.

$\mathrm{HCC}$ is one of the major causes of cancer-associated morbidity and mortality. HCC is the sixth most common cancer worldwide, being the fifth in men and the eighth in 

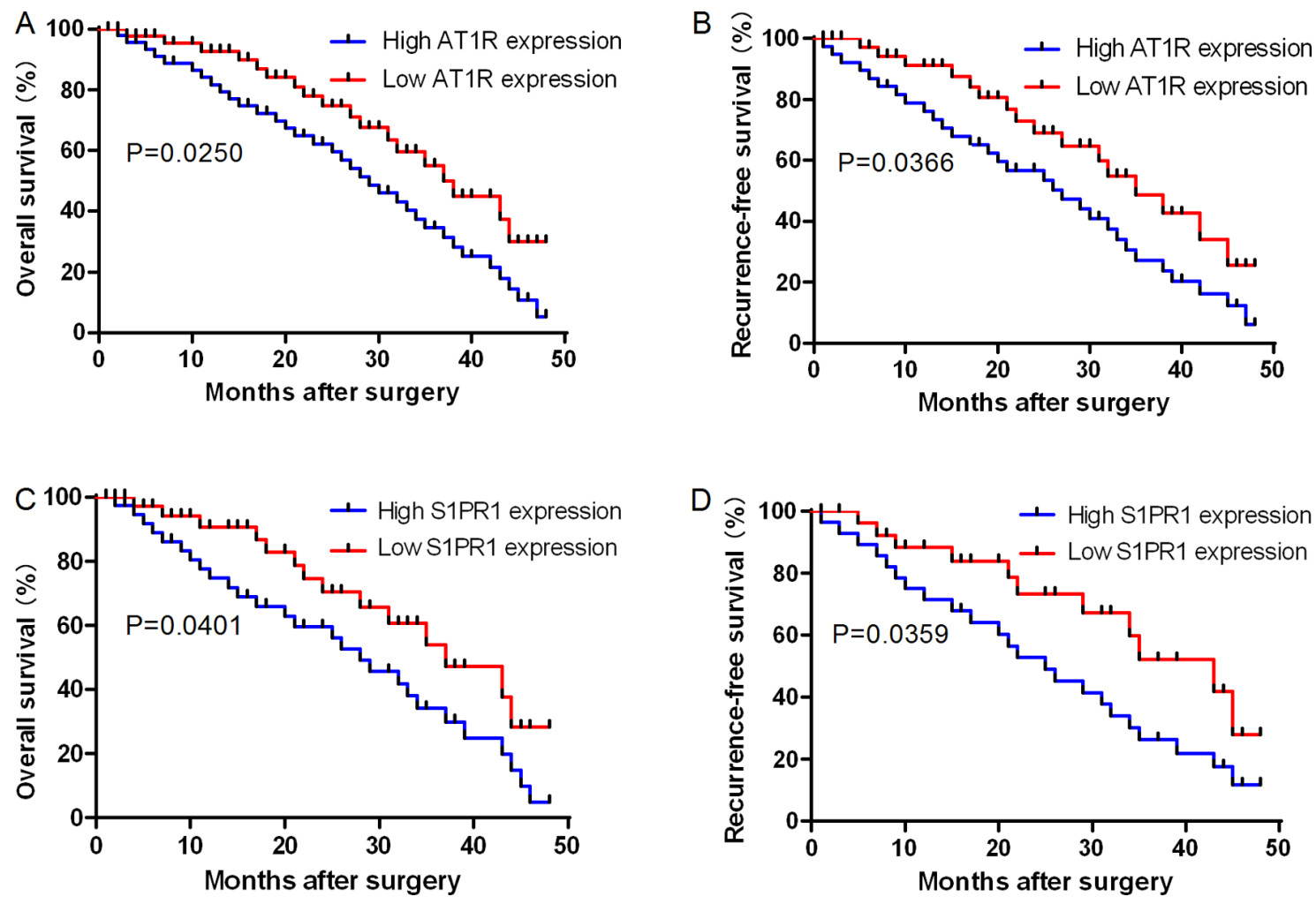

Figure 5. Kaplan-Meier survival analysis of patients with HCC stratified by AT1R and S1PR1 protein expression levels. (A) Overall and (B) recurrence-free survival rates of patients with high AT1R expression levels were significantly lower compared with those in patients with low AT1R expression levels. (C) Overall and (D) recurrence-free survival rates of patients with high S1PR1 expression levels were significantly lower compared with those in patients with low S1PR1 expression levels. HCC, hepatocellular carcinoma; AT1R, angiotensin II receptor type 1; S1PR1, sphingosine-1-phosphate receptor 1.

women, and accounting for $\sim 5.7 \%$ of all new cancer cases (35). Annually, around $1 \%$ of all deaths in the world are associated with HCC (35). Despite the development of novel diagnostic biomarkers, the OS rate of patients with HCC remains unsatisfactory. Thus, in the present study, the potential association between Ang II/S1P and AT1R/S1PR1 in HCC was investigated, and the roles of AT1R and S1PR1 protein expression levels in the progression and prognosis of HCC were examined. The results indicated that a higher serum Ang II level was correlated with a higher serum S1P level, and that higher AT1R expression levels were correlated with higher expression levels of S1PR1 in HCC tissue. Therefore, a positive correlation exists between Ang II and S1P, as well as between AT1R and S1PR1 in HCC.

Consistent with previous studies $(16,20)$, the present study demonstrated that Ang II and AT1R were associated with intrahepatic metastasis, portal vein invasion, tumor grade and clinical outcome. S1P serves a key role in the tumor by regulating cell survival, growth and invasion (36). In addition, S1P and S1PR1 overexpression in HCC have been associated with a poor prognosis $(37,38)$. The prognosis of patients with HCC remains unfavorable due to high recurrence and metastasis rates, and the 5-year OS rate of patients with $\mathrm{HCC}$ has been reported to be $16 \%(39,40)$. In the present study, the association between AT1R and S1PR1 protein expression levels and OS and RFS in patients with HCC was further investigated. AT1R and S1PR1 high expression was significantly associated with patient mortality and the recurrence of HCC. Notably, the 50-month survival rate of patients with high AT1R and
S1PR1 expression was low. Patients with high AT1R and S1PR1 protein expression levels exhibited poor outcomes with regard to OS and RFS compared with patients with low AT1R and S1PR1 expression levels, suggesting that AT1R and S1PR1 may serve as valuable prognostic biomarkers for patients with HCC.

In conclusion, the present study demonstrated that Ang II and S1P levels were elevated in the serum of patients with HCC, and that AT1R and S1PR1 mRNA and protein expression levels were also upregulated in HCC tumor tissue. Furthermore, the results demonstrated an association between AT1R and S1PR upregulation and HCC progression and pathological characteristics, including intrahepatic metastasis, portal vein invasion, TNM stage and histological differentiation via Edmondson grade analysis. In addition, a correlation between Ang II/S1P and AT1R/S1PR1 in HCC was also identified. The present study indicated that Ang II/AT1R and S1P/S1PR1 may be valuable indicators of a poor prognosis for patients with HCC.

\section{Acknowledgements}

Not applicable.

\section{Funding}

The present study was supported by the Natural Science Basic Research Program of Shaanxi (grant no. 2020JZ-41), the Key Research and Development Program of Shaanxi Province 
(grant no. 2017SF-313), the Fundamental Research Funds for the Central Universities of China (grant no. XJJ2017072) and the Personnel Training Specialized Research Foundation of the Second Affiliated Hospital of Xi'an Jiaotong University [grant no. RC(GG)201803].

\section{Availability of data and materials}

The datasets used and/or analyzed during the present study are available from the corresponding author on reasonable request.

\section{Authors' contributions}

YYJ and ZW conceived the study. YYJ performed the experiments and wrote the manuscript. YFJ, HC and WG analyzed data and provided experimental technical support. LM and YFJ performed the histopathological evaluation. ZW, ZY and $\mathrm{BH}$ interpreted results from a clinical perspective and analyzed the data. ZW helped to draft the manuscript. All authors read and approved the final manuscript.

\section{Ethics approval and consent to participate}

All of the experiments were approved by the Ethics Committee of the Second Affiliated Hospital of Xi'an Jiaotong University (approval no. 20160352; Xi'an, China). All patients provided written informed consent in accordance with the Declaration of Helsinki.

\section{Patient consent for publication}

Not applicable.

\section{Competing interests}

The authors declare that they have no competing interests.

\section{References}

1. Zhang X, Li J, Shen F and Lau WY: Significance of presence of microvascular invasion in specimens obtained after surgical treatment of hepatocellular carcinoma. J Gastroenterol Hepatol 33: 347-354, 2018.

2. El-Serag HB: Hepatocellular carcinoma: An epidemiologic view. J Clin Gastroenterol 35 (5 Suppl 2): S72-S78, 2002.

3. Dutta R and Mahato RI: Recent advances in hepatocellular carcinoma therapy. Pharmacol Ther 173: 106-117, 2017.

4. Bruix J, Reig M and Sherman M: Evidence-based diagnosis, staging, and treatment of patients with hepatocellular carcinoma. Gastroenterology 150: 835-853, 2016.

5. Maluccio M and Covey A: Recent progress in understanding, diagnosing, and treating hepatocellular carcinoma. CA Cancer J Clin 62: 394-399, 2012.

6. Dhir M, Melin AA, Douaiher J, Lin C, Zhen WK, Hussain SM, Geschwind JF, Doyle MB, Abou-Alfa GK and Are C: A Review and update of treatment options and controversies in the management of hepatocellular carcinoma. Ann Surg 263: 1112-1125, 2016.

7. Ogretmen B and Hannun YA: Biologically active sphingolipids in cancer pathogenesis and treatment. Nat Rev Cancer 4: 604-616, 2004.

8. Obinata $\mathrm{H}$ and Hla T: Sphingosine 1-phosphate and inflammation. Int Immunol 31: 617-625, 2019.

9. Kawamori T, Kaneshiro T, Okumura M, Maalouf S, Uflacker A, Bielawski J, Hannun YA and Obeid LM: Role for sphingosine kinase 1 in colon carcinogenesis. FASEB J 23: 405-414, 2009.
10. Go H, Kim PJ, Jeon YK, Cho YM, Kim K, Park BH and Ku JY: Sphingosine-1-phosphate receptor 1 (S1PR1) expression in non-muscle invasive urothelial carcinoma: Association with poor clinical outcome and potential therapeutic target. Eur J Cancer 51: 1937-1945, 2015.

11. Vrzalikova K, Ibrahim M, Vockerodt M, Perry T, Margielewska S, Lupino L, Nagy E, Soilleux E, Liebelt D, Hollows R, et al: S1PR1 drives a feedforward signalling loop to regulate BATF3 and the transcriptional programme of Hodgkin lymphoma cells Leukemia 32: 214-223, 2018.

12. Wang C, Mao J, Redfield S, Mo Y,Lage JM and Zhou X: Systemic distribution, subcellular localization and differential expression of sphingosine-1-phosphate receptors in benign and malignant human tissues. Exp Mol Pathol 97: 259-265, 2014.

13. Grammatikos G, Schoell N, Ferreirós N, Bon D, Herrmann E, Farnik H, Köberle V, Piiper A, Zeuzem S, Kronenberger B, et al: Serum sphingolipidomic analyses reveal an upregulation of C16-ceramide and sphingosine-1-phosphate in hepatocellular carcinoma. Oncotarget 7: 18095-18105, 2016.

14. Yoshiji H, Kuriyama S, Kawata M, Yoshii J, Ikenaka Y, Noguchi R, Nakatani T, Tsujinoue H and Fukui H: The angiotensin-I-converting enzyme inhibitor perindopril suppresses tumor growth and angiogenesis: Possible role of the vascular endothelial growth factor. Clin Cancer Res 7: 1073-1078, 2001.

15. Nakai Y, Isayama H, Ijichi H, Sasaki T, Sasahira N, Hirano K, Kogure H, Kawakubo K, Yagioka H, Yashima Y, et al: Inhibition of renin-angiotensin system affects prognosis of advanced pancreatic cancer receiving gemcitabine. Br J Cancer 103: 1644-1648, 2010.

16. Chowdhury A, Sarkar J, Pramanik PK, Chakraborti T and Chakraborti S: Cross talk between MMP2-Spm-Cer-S1P and ERK1/2 in proliferation of pulmonary artery smooth muscle cells under angiotensin II stimulation. Arch Biochem Biophys 603: 91-101, 2016

17. Tirapelli CR, Bonaventura D, Tirapelli LF and de Oliveira AM: Mechanisms underlying the vascular actions of endothelin 1, angiotensin II and bradykinin in the rat carotid. Pharmacology 84 : 111-126, 2009.

18. Dolley-Hitze T, Verhoest G, Jouan F, Le Pogamp P, Arlot-Bonnemains Y, Oger E, Belaud-Rotureau MA, Rioux-Leclercq $\mathrm{N}$ and Vigneau C: Angiotensin-2 type 1 receptors (AT1R) and cancers. Nephrol Ther 9: 85-91, 2013 (In French).

19. Ji Y, Wang Z, Li Z, Zhang A, Jin Y, Chen $H$ and Le $X$ : Angiotensin II enhances proliferation and inflammation through AT1/PKC/NF- $\kappa$ B signaling pathway in hepatocellular carcinoma cells. Cell Physiol Biochem 39: 13-32, 2016.

20. Hsu YC, Ho HJ, Wu MS, Lin JT and Wu CY: Postoperative peg-interferon plus ribavirin is associated with reduced recurrence of hepatitis $C$ virus-related hepatocellular carcinoma. Hepatology 58: 150-157, 2013.

21. Facciorusso A, Del Prete V, Crucinio N, Muscatiello N, Carr BI, Di Leo A and Barone M: Angiotensin receptor blockers improve survival outcomes after radiofrequency ablation in hepatocarcinoma patients. J Gastroenterol Hepatol 30: 1643-1650, 2015.

22. Lee DY, Won KJ, Lee KP, Jung SH, Baek S, Chung HW, Choi WS, Lee HM, Lee BH, Jeon BH and Kim B: Angiotensin II facilitates neointimal formation by increasing vascular smooth muscle cell migration: Involvement of APE/Ref-1-mediated overexpression of sphingosine-1-phosphate receptor 1. Toxicol Appl Pharmacol 347: 45-53, 2018.

23. Kanai T, Hirohashi S, Upton MP, Noguchi M, Kishi K, Makuuchi M, Yamasaki S, Hasegawa H, Takayasu K, Moriyama N, et al: Pathology of small hepatocellular carcinoma. A proposal for a new gross classification. Cancer 60: 810-819, 1987.

24. Edge SB and Compton CC: The American Joint Committee on Cancer: The 7th edition of the AJCC cancer staging manual and the future of TNM. Ann Surg Oncol 17: 1471-1474, 2010.

25. Zhang Y, Liu P, Jiang Y, Dou X, Yan J, Ma C, Fan Q, Wang W, Su F, Tang H and Su X: High expression of neuropilin-1 associates with unfavorable clinicopathological features in hepatocellular carcinoma. Pathol Oncol Res 22: 367-375, 2016.

26. Zhai X, Zhu H, Wang W, Zhang S, Zhang Y and Mao G: Abnormal expression of EMT-related proteins, S100A4, vimentin and E-cadherin, is correlated with clinicopathological features and prognosis in HCC. Med Oncol 31: 970, 2014.

27. Ji Y, Wang Z, Chen H, Zhang L, Zhuo F and Yang Q: Serum from chronic hepatitis $\mathrm{B}$ patients promotes growth and proliferation via the IGF-II/IGF-IR/MEK/ERK signaling pathway in hepatocellular carcinoma cells. Cell Physiol Biochem 47: 39-53, 2018. 
28. Livak KJ and Schmittgen TD: Analysis of relative gene expression data using real-time quantitative PCR and the 2(-Delta Delta C(T)) method. Methods 25: 402-408, 2001.

29. Abbott. Operations manual or user guide. Available from: https://www.corel abora tory.abbot t/regis trati on-ous/login.

30. Pyne NJ and Pyne S: Sphingosine 1-phosphate and cancer. Nat Rev Cancer 10: 489-503, 2010.

31. Pinter M, Weinmann A, Wörns MA, Hucke F, Bota S Marquardt JU, Duda DG, Jain RK, Galle PR, Trauner M, et al: Use of inhibitors of the renin-angiotensin system is associated with longer survival in patients with hepatocellular carcinoma. United European Gastroenterol J 5: 987-996, 2017.

32. Ye G, Qin Y, Lu X, Xu X, Xu S, Wu C, Wang X, Wang S and Pan D: The association of renin-angiotensin system genes with the progression of hepatocellular carcinoma. Biochem Biophys Res Commun 459: 18-23, 2015.

33. Xu ZW, Yan SX, Wu HX, Zhang Y and Wei W: Angiotensin II and tumor necrosis factor-alpha stimulate the growth, migration and invasion of BEL-7402 cells via down-regulation of GRK2 expression. Dig Liver Dis 51: 263-274, 2019.

34. Stoyanov E, Ludwig G, Mizrahi L, Olam D, Schnitzer-Perlman T, Tasika E, Sass G, Tiegs G, Jiang Y, Nie T, et al: Chronic liver inflammation modifies DNA methylation at the precancerous stage of murine hepatocarcinogenesis. Oncotarget 6: 11047-11060, 2015.

35. Kew MC: Epidemiology of chronic hepatitis B virus infection, hepatocellular carcinoma, and hepatitis B virus-induced hepatocellular carcinoma. Pathol Biol (Paris) 58: 273-277, 2010 .
36. Pyne NJ, Ohotski J, Bittman R and Pyne S: The role of sphingosine 1-phosphate in inflammation and cancer. Adv Biol Regul 54: 121-129, 2014

37. Bao M, Chen Z, Xu Y, Zhao Y, Zha R, Huang S, Liu L, Chen T, $\mathrm{Li} \mathrm{J}, \mathrm{Tu} \mathrm{H}$ and $\mathrm{He} \mathrm{X}$ : Sphingosine kinase 1 promotes tumour cell migration and invasion via the S1P/EDG1 axis in hepatocellular carcinoma. Liver Int 32: 331-338, 2012.

38. Matsushima-Nishiwaki R, Yamada N, Fukuchi K and Kozawa O: Sphingosine 1-phosphate (S1P) reduces hepatocyte growth factor-induced migration of hepatocellular carcinoma cells via S1P receptor 2. PLoS One 13: e0209050, 2018.

39. Fong ZV and Tanabe KK: The clinical management of hepatocellular carcinoma in the United States, Europe, and Asia: A comprehensive and evidence-based comparison and review. Cancer 120: 2824-2838, 2014.

40. Wong RJ, Devaki P, Nguyen L, Cheung R and Nguyen MH: Ethnic disparities and liver transplantation rates in hepatocellular carcinoma patients in the recent era: Results from the Surveillance, Epidemiology, and End Results registry. Liver Transpl 20: 528-535, 2014.

This work is licensed under a Creative Commons Attribution-NonCommercial-NoDerivatives 4.0 International (CC BY-NC-ND 4.0) License. 